\title{
Design and implementation of low coupling and high available mobile workflow for power operation
}

\author{
Gang Wang ${ }^{1,}$ a, Xingchuan Bao ${ }^{1, b}$, Min $\mathrm{Xu}^{1, \mathrm{c}}$, Zhansheng Hou ${ }^{1, \mathrm{~d}}$, Haiyun \\ $\operatorname{Han}^{1, e}$, Lin Peng ${ }^{1, f}$
}

${ }^{1}$ State Grid Smart Grid Research Institute, Nanjing 211106, China

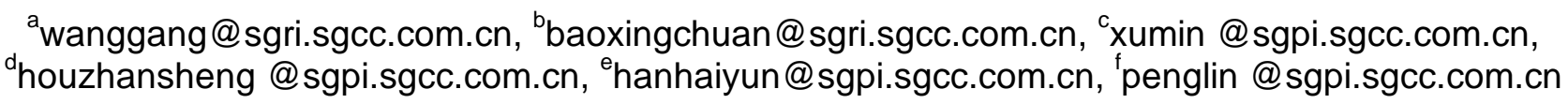

Keywords: mobile workflow; power mobile operation.

Abstract. In the field of the power supply enterprise, it is urgent to use the mobile workflow to carry out the process management. This paper adopts low coupling and high available workflow design, which design the workflow client through the long connection and SMS with the power mobile operation state manager collaborative managing workflow task and keeping the mobile workflow running in the poor communication condition. Power mobile operation client state management and workflow execution collaboration manage mobile workflow tasks, which achieve high available workflow based on the low cost.

\section{Introduction}

Currently there are many problems in the power supply enterprise standardization job. There are many standardized work instructions, cards, maintenance records, test report types and different, harder to correspond work tickets. This model has many drawbacks, such as the inability to of in-situ flow management, information communication needing telephone communication, needing a lot of preparatory work before operation. With the maturity of mobile technology and mobile terminal processing ability more and more powerful, along with the development of mobile communication technology, it is necessary to study the power of mobile operation of low coupling high availability workflow design method, meeting needs of power shift operation.

Taking into account many of the transmission line inspection operations are in remote mountainous areas, the communication rate is relatively low, and even can only meet the requirements of SMS. But it needs high availability communication in power mobile operating. Mobile operation function is complex, needing low coupling and high available power mobile operation process. At present, most workflow software only supports online Interne and lack of support of mobile communication operation. So, electric mobile low coupling high availability workflow design is particularly important.

The traditional workflow software contains the workflow definition, workflow driven and workflow management $\mathbf{I}^{\mathbf{1}-3} \mathbf{I}$, but not for mobile environment, needs to provide low coupling and high availability workflow design method.

\section{Mobile workflow application analysis}

With the maturity of mobile technology and mobile terminal processing ability more and more powerful, along with the development of mobile communication technology, more and more companies are expanding business to mobile, and mobile devices into the enterprise business has become an important trends. Mobile applications gradually become the preferred mode for users. In this technology and business trends, mobile platform become the basic platform of IT construction, help the enterprise to realize the fast business mobile. It has been used in power grid enterprise mobile application, such as equipment inspection, equipment maintenance, equipment testing, equipment testing in production domain, mobile meter reading, power inspection in marketing business. 
Most of the workflow management in power mobile applications is coupled with business ${ }^{\mathbf{I}^{4-5} \mathbf{I}}$. Workflow application is not much. Most of the work tasks are offline and cannot support workflow in real time.

Mobile operation workflow provide remote access of mobile client application. The user in the outdoor environment use the remote workflow assistance to facilitate the work, and use resources required in site. The workflow module in the server includes the mobile client authentication, the workflow driver and the workflow management. Through the coordination of other modules, it completes the information exchange between the scene video, voice and other information, and supports remote expert assistance.

\section{Mobile workflow architecture design}

In order to meet the needs of existing mobile workflow technology, mobile workflow platform needs to provide the heterogeneous network access, mobile client processing workflow, mobile client authentication, workflow engine driver, mobile operation workflow definition, and the workflow management.

The specific workflow design is as follows: a low coupling and high availability workflow for power operation is designed. The technical proposal is adopted as follows: workflow process definition defined mobile workflow, mobile workflow actuator executed workflow, the workflow execution in power mobile terminal accepts the execution of workflow and feedbacks the workflow state, the operation of electric mobile client state manager manage workflow execution state on the client.

Power mobile client state manager uses two ways to manage the workflow: The first method is to manage the workflow execution client token connection by TCP long connection mode. The state of the client task state is monitored by token. And the state is synchronized to the workflow process. Second, through the SMS method, the client state manager executes the client message, extracts the mobile work tasks, and synchronizes state to the workflow process.

Workflow execution client uses two ways to implement the workflow: The first method is to create a unique token task by TCP long connection and to perform user tasks through token. Second, through the SMS way, workflow execution client resolve the power mobile client state server packet and receives the user tasks.

Workflow process definition can define parallel, serial model of mobile workflow and define workflow execution roles. Workflow process execution is responsible for executing workflow process definition, which and power mobile client state manager, workflow execution client co drive process.

The following diagram is the workflow state interaction diagram:

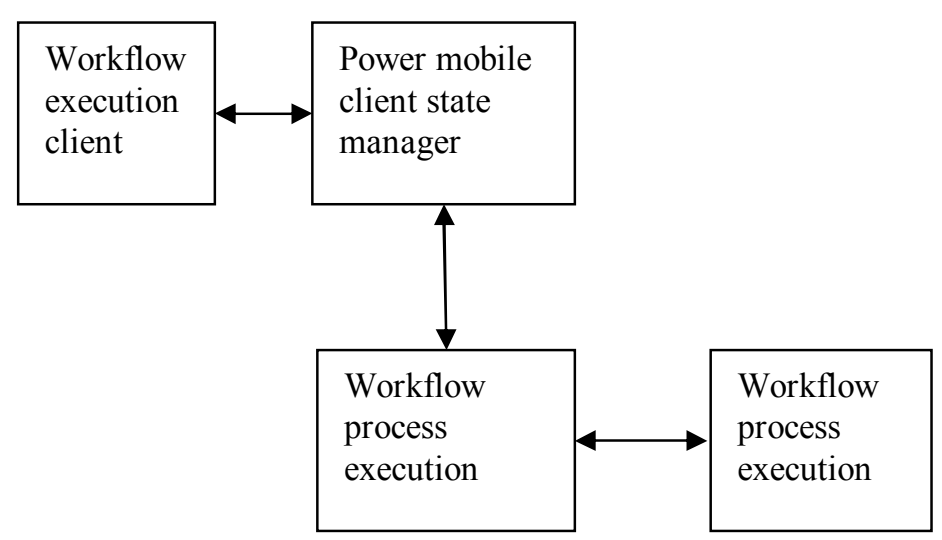

Fig. 1 workflow state interaction diagram 


\section{Function design and Implementation}

\section{A. Functional architecture}

For low coupling high availability of workflow used in the power mobile operation, the system must provide mobile operation workflow client management, mobile workflow driving, workflow processing and workflow management. Compared to the common workflow, the biggest difference of the mobile workflow is that the outdoor communication conditions is poor and workflow must be designed to maintain real-time online state to accomplish the task. Mobile workflow communication is based on $2 \mathrm{G} / 3 \mathrm{G} / 4 \mathrm{G}$ and SMS to complete information transmission. Workflow tasks must be properly resolved. Workflow engine also needs to take into account the state management of workflow. Workflow needs to provide a mechanism for correcting errors in execution. Workflow also needs to provide interface to query information for external program. The following diagram is a functional diagram of mobile workflow design.

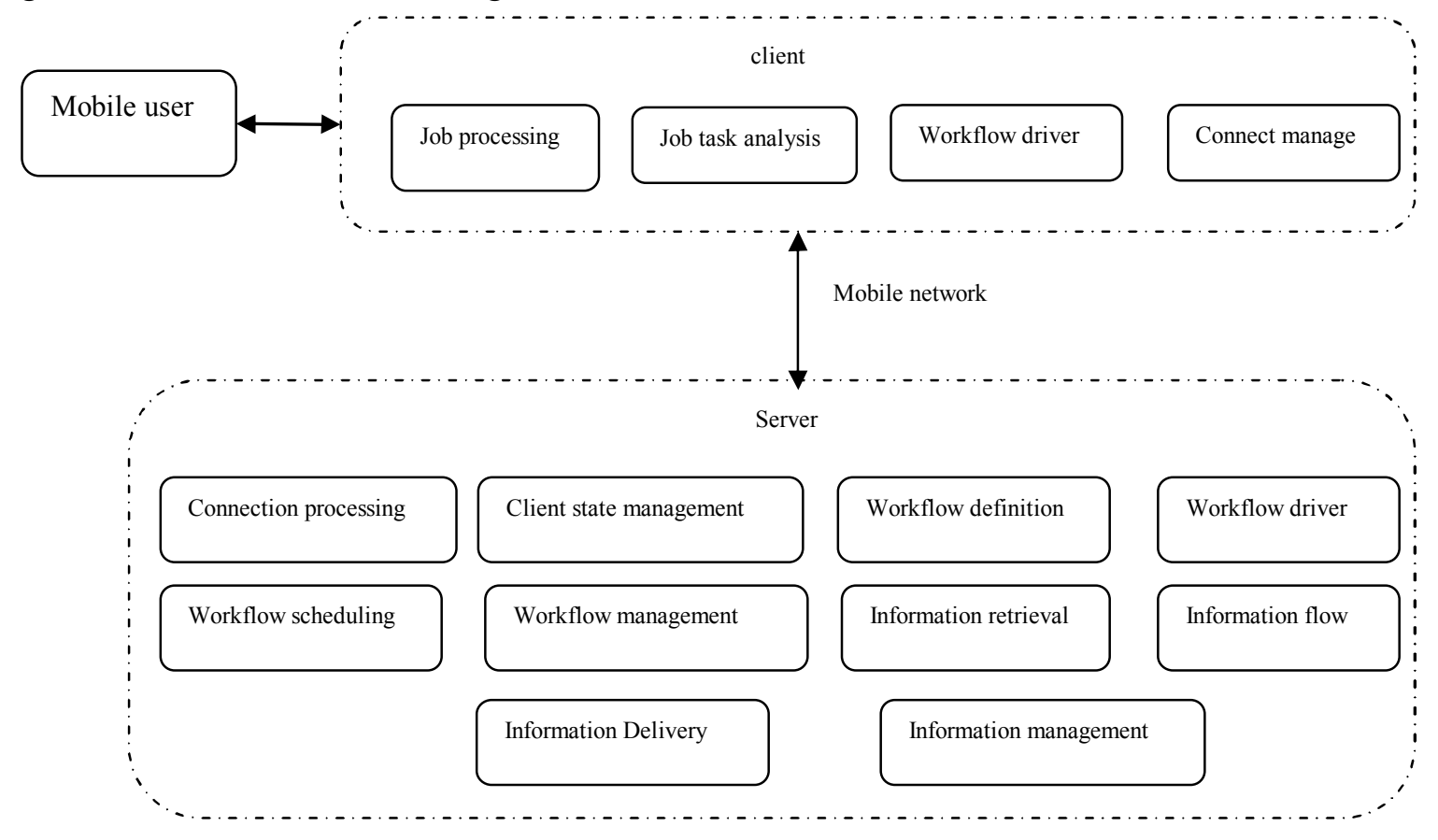

Fig. 2 functional diagram of mobile workflow

Workflow system functions is included in the mobile client and server. Functions in the mobile client include job processing, job task analysis, workflow driver, connect manage. The main purpose of the client is to accept the server workflow task, responsible for the interpretation of the implementation of the workflow task and the transfer of information to the user, responsible for receiving the user to perform workflow information and passing information to the server. Functions in the server include connection processing, client state management, workflow definition, workflow driver, and so on. The main purpose of the server is to explain and execute the workflow.

\section{B. System architecture}

Mobile workflow in power operation optimizes the current J2EE architecture. The multi-tier architecture model is based on Andriod/IOS+J2EE.

Display layer: technology using Andriod/IOS client provides a full range of workflow experience for the user.

Service layer: J2EE encapsulates numerous business logic, and provides Andriod/IOS remote method call and service interface for Andriod/IOS layer to call the JAVA service layer to complete the page display function. 


\begin{tabular}{|c|}
\hline Andriod/ IOS client ( html5,css $3, \mathrm{js})$ \\
\hline Mobile application page \\
\hline Application package \\
\hline Service mapping layer \\
\hline JAVA service \\
\hline J2EEserver \\
\hline Database \\
\hline
\end{tabular}

Fig. 3 Architecture diagram

\section{Conclusions}

In this paper, the workflow execution client through long connection and SMS with the power of mobile operation state on the client manage collaborative workflow, which avoid the problem that mobile workflow in the poor communication can't be maintained. The design that the power mobile client state manager, workflow process execution collaborative manage mobile workflow task realizes low cost and high available workflow. Workflow in the pager can achieve the effective operation of the mobile operation in different communication environment, effectively supporting the power mobile site operation process.

\section{References}

[1] Leymann F. Workflow-based Applications. IBM Systems Journal,1997,36(1):102 122.

[2] Zhanghua,Wangxi. Research and implementation of service oriented workflow compensation mechanism [J]. Journal of Southeast University, 2009, 39(1): 40-46.

[3] Zhiyong L. Relation-based lightweight workflow engine[J]. Computer Applications and Software, 2005, 4: $53 \sim 57$.

[4] LinFeng, LinYi, HuJun. The Design and Implementation of the Platform Supporting General WorkFlow. Automation of Electric PowerSystems , 1999 , 23(13):45 47.

[5] Zhengwei,Yanbaoping. Review of workflow model [J]. Computer application and research, 2005, 22(5):11-13. 